\title{
BMJ Open Doctors' perspectives on PSA testing illuminate established differences in prostate cancer screening rates between Australia and the UK: a qualitative study
}

\author{
Kristen Pickles, ${ }^{1}$ Stacy M Carter, ${ }^{1}$ Lucie Rychetnik, ${ }^{2}$ Vikki A Entwistle ${ }^{3}$
}

To cite: Pickles K, Carter SM, Rychetnik L, et al. Doctors' perspectives on PSA testing illuminate established differences in prostate cancer screening rates

between Australia and the UK: a qualitative study. BMJ Open 2016;6:e011932. doi:10.1136/bmjopen-2016011932

- Prepublication history for this paper is available online. To view these files please visit the journal online (http://dx.doi.org/10.1136/ bmjopen-2016-011932).

Received 16 March 2016 Revised 4 November 2016 Accepted 9 November 2016

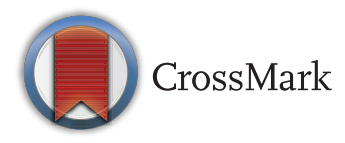

${ }^{1}$ Centre for Values, Ethics and the Law in Medicine, University of Sydney, Sydney, New South Wales, Australia

${ }^{2}$ School of Medicine, University of Notre Dame, Sydney, New South Wales, Australia

${ }^{3}$ Health Services Research Unit, University of Aberdeen, Foresterhill Aberdeen, UK

Correspondence to Kristen Pickles; kristen.pickles@sydney. edu.au

\section{ABSTRACT}

Objectives: To examine how general practitioners (GPs) in the UK and GPs in Australia explain their prostate-specific antigen (PSA) testing practices and to illuminate how these explanations are similar and how they are different.

Design: A grounded theory study.

Setting: Primary care practices in Australia and the UK. Participants: 69 GPs in Australia $(n=40)$ and the UK $(n=29)$. We included GPs of varying ages, sex, clinical experience and patient populations. All GPs interested in participating in the study were included.

Results: GPs' accounts revealed fundamental differences in whether and how prostate cancer screening occurred in their practice and in the broader context within which they operate. The history of prostate screening policy, organisational structures and funding models appeared to drive more prostate screening in Australia and less in the UK. In Australia, screening processes and decisions were mostly at the discretion of individual clinicians, and varied considerably, whereas the accounts of UK GPs clearly reflected a consistent, organisationally embedded approach based on local evidence-based recommendations to discourage screening.

Conclusions: The GP accounts suggested that healthcare systems, including historical and current organisational and funding structures and rules, collectively contribute to how and why clinicians use the PSA test and play a significant role in creating the mindlines that GPs employ in their clinic. Australia's recently released consensus guidelines may support more streamlined and consistent care. However, if GP mindlines and thus routine practice in Australia are to shift, to ultimately reduce unnecessary or harmful prostate screening, it is likely that other important drivers at all levels of the screening process will need to be addressed.

\section{BACKGROUND}

Prostate-specific antigen (PSA) testing of asymptomatic men for prostate cancer risk is

\section{Strengths and limitations of this study}

- The published literature about prostate cancer screening in general practice is replete with quantitative studies: They have identified clinician and patient demographics associated with more or less screening, and have analysed rates of screening and frequency of discussions about prostate-specific antigen (PSA) testing. Our research is complementary to existing quantitative findings: We asked how PSA testing of asymptomatic men occurs in clinical practice, from the general practitioner (GP) perspective.

- This study applied grounded theory methodology to explore how clinicians describe their prostate screening practice, and relate these to points of variation in the respective healthcare systems. Grounded theory is a suitable approach for the investigation of complex multifaceted processes (like PSA screening) occurring in context.

- We interviewed a large number of highly informative participants (GPs) in Australia and the UK with diverse opinions and approaches.

a contested issue internationally. In this paper, 'asymptomatic' will refer to those men attending clinical practice with no prior indications associated with prostatic disease. This is in contrast to the detection of prostate cancer in symptomatic men: men who have symptoms that could be related to locally advanced or metastatic prostate cancer such as frequency of urination, new onset bone pain and/or neurological symptoms involving the lower extremities. ${ }^{1}$ PSA testing of asymptomatic men is not recommended as a population-screening programme in Australia or the UK, the two countries on which our analysis focuses.

Prostate cancer incidence varies more than 25-fold worldwide. Incidence figures, which incorporate both life-saving diagnoses and 
overdiagnosed cancers, ${ }^{2}$ vary between Australia, where incidence is 115.22 cases per 100000 population, and the UK, where incidence is 73.19 per 100000 . Despite this difference in reported incidence, the two countries have roughly equivalent prostate cancer mortality figures: 12.88 and 13.07 per 100000 , respectively. ${ }^{3}$ There are many reasons for variation in incidence and mortality rates, which could be due to underlying differences in prostate cancer risk and population age structures, men presenting for testing, access and availability of treatment options, cancer coding and registration and diagnostic processes (such as availability of PSA testing and improved diagnosis).

An important factor influencing reported prostate cancer incidence in a population is PSA testing rates: higher testing rates produce higher incidence. Testing rates may be influenced by differences in professional and organisational policies, media, cultural beliefs and values. Annual PSA testing rates of asymptomatic men in general practice are difficult to ascertain. The data collected often do not distinguish men who have had a PSA for prostate cancer screening from those in whom established disease is being monitored.

Clinical practice in the UK and Australia is grounded in the same evidence base and international literature, yet the two jurisdictions have notably different rates of PSA testing. In the UK, a study in six English cities reported the annual practice-based PSA testing rate for 2007 (in men aged $45-89$ years) to be $6.2 \% .^{4}$ A more recent study analysed data from patient electronic records in primary care for men aged $45-84$ years. It reported that for every 100 men enrolled with a general practitioner (GP) for one year, 5.03 (asymptomatic men) were tested in 2010 , and the rate increased by $8 \%$ in 2011 to 5.45 per $100 .^{5}$ Note that the data this analysis was based on represent only $5 \%$ of the population in England and may not be representative of all practices.

Analysis of Medicare Australia's Medical Benefits Scheme records suggests that each year at least $20 \%$ of men aged 45-74 years have a PSA test, presumably for the purpose of early diagnosis of prostate cancer. ${ }^{6}$ This number underestimates the actual number of PSA tests performed, by up to $40 \% .^{7}$ The prevalence of PSA testing in men over 50 years in Australia was reported at $63 \%$ in 2003; that is, $63 \%$ of men $>50$ years had ever had a PSA test. ${ }^{8}$ This proportion is likely to have risen since. ${ }^{9}$

This raises questions about how practice in the two jurisdictions, while drawing on the same evidence base, could be so markedly different. In table 1 , we set out some important differences in the organisation and funding of primary healthcare between Australia and the UK, including subtle variance in the advice offered to GPs from expert authorities in relation to how actively physicians should offer testing. Any or all of these differences conceivably influence testing rates.

Here, we report on a comparative qualitative study of Australian and UK GPs' current approaches to, and reasoning about, PSA testing of asymptomatic men to address the following questions:
- How do GPs in the UK and GPs in Australia explain their PSA testing practices?

- How are these explanations similar and different?

Our analysis draws on Gabbay and le May's ${ }^{14}$ concept of 'mindlines', which they developed to explain how GPs use research evidence in practice. They describe mindlines as 'collectively reinforced, internalised, tacit guidelines', ${ }^{15}$ mainly grown and refined via training, experience and interaction with trusted sources, and mediated by features of primary care organisations, including their ethos and financial and structural elements. Their mindlines theory makes a valuable contribution to the evidence-based medicine (EBM) and knowledge translation literatures because the theory considers the influence of local context on GP adherence and use of formal knowledge like guidelines. We use our analysis of data collected from GPs practising in Australia and the UK to extend Gabbay and le May's theory of mindlines.

\section{METHODS \\ Design}

We applied the well-established, systematic qualitative research methodology of grounded theory. ${ }^{15}$ Grounded theory is a method of conducting qualitative research that focuses on creating conceptual frameworks or theories through building inductive analysis from the data. ${ }^{16}$ Grounded theorists are led by the experiences of the people in their inquiry and the substantive theories they develop closely reflect what those people experience and do. Specific methods of data collection and analysis are used to identify patterns in the research data. The twin foundations of grounded theory are the processes of constant comparison (a simultaneous and concurrent process of coding and analysis) and theoretical sampling (sampling with the aim of developing the properties of a developing idea or theory). These methods together guide the systematic development of emerging theory, and ensure findings remain firmly grounded in the collected data. All study authors have been formally trained in the methods described; SC has particular expertise in grounded theory methodology.

\section{Participants and setting}

We purposively recruited a sample of 69 GPs (Australia, $\mathrm{n}=40$; the UK, $\mathrm{n}=29$ ) for this study. In Australia, we advertised via the newsletters and email lists of GP organisations (Medicare Locals) in Sydney, and in mass and social media, and in medical journals. As analysis and sampling evolved, we invited additional rural and interstate GPs to answer specific analytical questions; for example, the influence of GP proximity to specialist services. Rural GPs were accessed by phoning practice managers, through colleagues, and advertising with rural Medicare Locals. ${ }^{17}$ When we encountered GPs whose routine care was divergent from previously interviewed GP norms, we invited more GPs from that practice to 
Table 1 A comparison of Australian and UK health systems and PSA testing context

Australia The UK

How is primary

healthcare provided?

Are GPs advised to offer PSA testing?

How should men be tested (if they choose to be tested)?
The Australian Medicare system is predominantly based around private practice and fee-for-service funding, that is, private practitioners in independent businesses are paid for each instance of service, mainly using public funds through the MBS, sometimes supplemented by patient copayments. Some GPs bulk bill, that is, GPs charge the Government (Medicare) directly for any medical service that their patient receives. In these practices, GPs receive the Medicare rebate (a fixed sum for each type of service) as payment, and patients pay nothing. There is considerable geographical variation in bulk billing practice depending on where the GP is based (less in more affluent areas and in rural, regional and remote areas, ${ }^{10-12}$ where there is a greater shortage of doctors and healthcare services). There are standards but GPs are mostly free to set their own fees for consultations and procedures. Some charge substantially more than the value of the Medicare rebate.

Australians can consult any GP they choose, including seeing multiple GPs in multiple practices.

RACGP advises its members not to raise the issue of PSA testing with patients, but if men ask, to fully inform them about the potential benefits, risks and uncertainties.

USANZ advises for men at average risk of prostate cancer, who have been informed of the benefits and harms of testing and who decide to undergo regular testing for prostate cancer, offer PSA testing every 2 years from $50-69$ years, and offer further investigation if total PSA is greater than $3.0 \mathrm{ng} / \mathrm{mL}$.

GPs advised to discuss the pros and cons of testing with eligible men.

2012 RACGP Red Book guidance advises GPs to perform PSA and DRE when a patient chooses screening, whereas Australian NHMRC guidelines do not recommend DRE for asymptomatic men in the primary care setting. USANZ suggests that GPS confident in performing DRE are still encouraged to do so.
The countries of the UK have centralised health systems-the NHS. General practices mostly operate as independent businesses managed by GPs delivering care under contract to the NHS and free to the patient at point of use. GPs receive some payment on a capitation basis (practices receive a fixed amount to manage a set of potential patients).

There is virtually no fee for service element, but some of the money practices receive from the NHS is dependent on them supplying evidence that certain quality standards have been met (eg, that at least $n \%$ of people with a diagnosis of $x$ have received intervention $y$ ).

Almost all residents in the UK are registered to a GP practice near their usual home, and will consult GPs within that practice.

Universal screening for prostate cancer is not recommended; however, PSA testing can be provided at patient request (UK National Screening Committee).

EAU advises that informed men requesting an early diagnosis should be given a PSA test and undergo a DRE. A risk-adapted strategy might be considered based on the initial PSA level.

GPs advised to discuss the pros and cons of testing with eligible men.

DRE is not recommended as a screening test in asymptomatic men in the UK (NHS PCRMP). Men aged $>50$ years who request a PSA test can access the NHS PCRMP, an informed choice programme introduced by the Government in 2002 to ensure that men concerned about prostate cancer receive clear and balanced information. The PCRMP provides GPs with information to counsel any man who asks about PSA testing. It is an English initiative, but Wales, Scotland and Northern Ireland have adopted the same approach and use the same materials PSA testing can be provided free on the NHS for men over 50 years on the condition they have made an 'informed choice' following a GP consultation. tests for men 50 years and over since 1989 through the MBS. ${ }^{13}$

Is the PSA test funded?

DRE, digital rectal examination; EAU, European Association of Urology; GP, general practitioner; MBS, Medicare Benefits Schedule; NHMRC, National Health and Medical Research Council; NHS, National Health Service; NHS PCRMP, National Health Service Prostate Cancer Risk Management Programme; PSA, prostate-specific antigen (test); RACGP, Royal Australian College of General Practitioners; USANZ, Urological Society of Australia and New Zealand. 
attempt to distinguish between personal and institutional influences on their practice.

We recruited 29 GPs throughout England ( $n=23)$, Scotland $(n=5)$ and Wales $(n=1)$ to explore PSA testing reasoning and practice in a jurisdiction with comparatively lower rates of prostate cancer screening than Australia. The initial sample of GPs responded to an invitation distributed by academic colleagues through professional networks. We then broadened the sample by advertising via email to members of the Royal College of General Practitioners (RCGP), primary healthcare departments, university academic departments and general practice and research mail lists. We also advertised via newsletter including the Society for Academic Primary Care and RCGP Scotland's eBulletin.

GPs were invited to contact KP if they were interested and willing to participate. An information sheet outlining the research project was emailed to all respondents. Participants were of varying ages, clinical experience, sex and patient populations: all GPs who expressed interest in participating were included. GPs were compensated for their time.

\section{Interviews/data collection}

We generated data via in-depth interviews. The semistructured interview schedule covered a broad range of topics, including GPs' recent clinical encounters involving PSA testing decisions, communicating information about PSA testing to patients, screening pathways for PSA and overdiagnosis of prostate cancer. The schedule was modified between interviews based on the developing analysis to enrich the data available to answer our research questions. All GPs were asked to think back to their most recent consultation involving a discussion about PSA testing or to describe a typical consultation where the topic was raised. The aim of this approach was to open the discussion about, and provide context for, conversations about PSA testing and to use as a platform to guide prompts and to focus subsequent questions.

Interviews took place between March 2013 and June 2014 (Australian GPs) and between September and December 2014 (UK GPs). We continued to interview GPs until we judged we had reached theoretical saturation, that is, the point at which gathering more data ceases to yield any further insights about the emerging grounded theory. All interviews were conducted by KP, primarily by telephone or Skype, and ranged in duration from 18 to $70 \mathrm{~min}$. All interviews were audio-recorded, and were transcribed verbatim.

\section{Data coding and analysis}

The analysis was led by KP, who coded the transcripts and wrote detailed memos which were reviewed and discussed by the authors in analysis meetings. A subset of transcripts was read and coded by three authors independently; this coding was compared and discussed to inform the development of the central concepts in the study. All concepts were derived directly from the data. Transcripts were not returned to participants for comment; all participants will receive a written summary of the research findings on study completion.

\section{Role of the funding source}

This study was funded by Australia's National Health and Medical Research Council (NHMRC) through a peer-reviewed competitive process. The funder had no role in the design, conduct or reporting of the study.

\section{RESULTS}

We identified notable differences in GPs' explanations of PSA testing in their individual practices, and within GPs' descriptions about the conditions of the respective health systems in Australia and the UK. One striking difference was that Australian GPs reported that they frequently spoke with asymptomatic men about being screened for prostate cancer, while UK GPs reported that they did this rarely. Another was that the process by which PSA testing occurred in each country appeared to be quite different. We explore below potential explanations for these differences.

\section{How did UK and Australian GPs' descriptions of their practices differ? \\ Testing as an exception versus testing as routine}

GPs' descriptions of a 'typical' consultation with a PSA-age man were very different in the two jurisdictions. Most GPs practising in the UK commented that PSA testing is quite uncommon and is certainly not considered routine practice. GPs said it would be 'rare' or 'unusual' for asymptomatic men to request PSA testing. UK GPs noted they may only receive a few PSA requests a year and some could not recall an example to refer to in the interview. The idea that a GP might introduce PSA testing as an issue for consideration for an otherwise healthy man was seen as strange; "if they're coming in with other issues, then we wouldn't say, oh by the way, you don't want a PSA test, do you? That just wouldn't happen" (GP1). UK GPs tended not to order the test unless specifically asked to do so, and some would "work quite hard to talk [asymptomatic] people out of it" (GP12).

In stark contrast, many Australian GPs talked about PSA testing as an everyday, usual part of consultations, and all reported that they commonly received requests for the test. Some said that on a typical day, they ordered several PSA tests for asymptomatic men. A significant proportion of the Australian GPs interviewed said that they might also raise PSA testing with a patient, unprompted. They reported that they would suggest testing because of a man's age, or raise it in the context of a health check. To quote one GP: "people are used to sort of being screened... so we're tacking this onto the discussion basically" (AGP21). Some GPs said their patients "don't get a chance" to initiate a discussion about PSA testing before the GP "talks them into it there and then" (AGP29). 
How PSA testing occurred: immediate testing versus a cooling-off period with extra information

The main difference between UK and Australian GPs' descriptions of how PSA testing occurred was that for Australian men, a decision about testing, and the ordering of a PSA test, was likely to occur in the consultation in which it was first raised. In contrast UK GPs described a two-step process, with a 'cooling-off' period between discussion and testing (if testing occurred at all).

UK GPs consistently reported the use of written information leaflets (mostly from www.patient.co.uk). They would give these to men who asked about PSA testing to take away and read to help them decide whether they wanted to be tested. GPs said that verbal discussions within the consultations were relatively brief because of the comprehensiveness of this resource. GPs noted that having a built-in 'cooling-off period' effectively (1) demonstrates that PSA testing is not something to rush into, and (2) allows patients to absorb the information in the leaflet before making a decision. A number of GPs said that men decided not to have PSA testing after receiving the information, "but whether that is because they've really understood the information or whether they've just picked up on the vibe, I couldn't really give you a good answer on that' (GP15).

GPs in Australia did not commonly report providing written information to their patients; they predominantly described having a verbal discussion only. Some GPs described engaging men in quite a detailed explanation about PSA testing, while other GPs said their discussion was "very, very brief" (AGP14). One GP, for example, reported: 'I give them next to none [information]. I say 'Do you want to find out if you've got prostate cancer?" (AGP9). Sometimes there was no discussion prior to testing. Other GPs apparently tried-and said they would sometimes succeed-to counsel men out of having a PSA test, reporting, for example, that "nine out of ten will choose not to have the test after appropriate explanation" (AGP23). These examples illustrate the considerable diversity of practice in Australia compared with the relative consistency of practice in the UK.

\section{What practice conditions did GPs report that might help explain differences in practice?}

We explored GPs' descriptions of training, structures and availability of resources, which served as anchors for their use of PSA testing as a screening tool. We outlined the similarities and differences we observed in UK and Australian GPs' accounts of the conditions under which they practiced. Table 2 is a representation of the 'system': not just the health system but the broader social and funding structures, comprising several interacting components.

Figure 1 illustrates the direction in which the system factors presented in table 2 potentially influence prostate screening: towards more PSA testing or towards less PSA testing, and including those system factors with no reported impact on testing rates.

Here, we present three overarching themes we subsequently identified as underlying and likely contributing to the variation evident in the GP's accounts: (1) history of PSA testing policy, (2) healthcare structures and incentives and (3) GP's preferred source of knowledge.

The history of PSA testing policy in the two countries is an important distinguishing factor

A number of GPs in the UK reported the long-standing consistency of a central authoritative position discouraging prostate cancer screening. The policy could be summarised as 'don't raise it, and inform if asked'. From the mid-1990s all GPs in the UK were sent relatively easy-to-read summaries of the evidence together with written information to hand out to men who asked about the PSA test. ${ }^{18}$ Dissemination of similar information has been used continuously since.

In contrast, in the Australian context, some GPs recalled the positive publicity that accompanied PSA testing when it was first introduced. Online documents indicate that, simultaneously, some Australian authorities actively encouraged and promoted PSA testing of asymptomatic men, while other official guidelines, released as early as 1995, recommended against prostate screening. ${ }^{19} 20$ As shown in table 2, Australian GPs reported that the guideline environment is challenging to navigate, making it difficult for GPs to find consistent and centrally issued directive advice.

The early messaging to GPs seems to have had longlasting implications. GPs in both countries commented on the tendency to continue practising in the manner in which they began. One UK GP explained, "it's quite difficult to change your practice. I think if you're a GP who's never been doing the PSA test then it's easier for you to carry on not doing them" (GP9). An Australian GP said,

I guess we learn from our initial experiences and it's very hard to change your initial thoughts on a particular test. So my initial understanding was it's an amazing test, that it should be done, that it's very useful, that you're almost neglectful not to do it... the initial thing was PSA is useful and that has basically stuck in my head, that PSA testing is useful. (AGP1)

\section{How healthcare structures and incentives drive practice}

The clinicians' accounts clearly reflected the known differences in healthcare payment models between the two locations. In Australia, GPs are paid fee-for-service-a scheme dependent on the quantity of instances of patient care-thus more patients, procedures and appointments generate greater income for GPs. One GP commented on the implications of fee-for-service,

If I went around having my 10 minute discussion with all my patients about why not to do PSA testing, I will make less money than [a GP] who does the 30 second-here Jack, that's a good idea, here, have the PSA test, we'll see you in a fortnight to check the result... one of the consequences of the current health system is that it feeds itself to making more diagnoses and being more busy, not less busy. (AGP23) 


\section{Situation in the UK}

Prostate Doctors screening healthy people, or healthy

screening culture people demanding this, considered strange; 'it doesn't happen. People don't come in and say

"they feel fine, they just want all their blood checks"...I don't think the NHS could really do that' (GP21).

GP training

UK GPs felt trained to avoid PSA testing, a 'really big topic' and 'classic case' in medical exams and training. "The training that we received is ... how you would have a discussion ... when we're asked for [the test], and you almost felt like they were sort of trying to dissuade asymptomatic men from having it.. that was definitely the sort of slant' (GP16).

Funding models UK GPs spaced appointments to allow for appropriate care, because "the expectation from the UK government as purchasers of the care would be that [men] be counselled around the limitations" (GP15). They were acutely aware of "a responsibility [for] spending the finite [NHS] resources" (GP23). UK GPs and practices did not gain financially from test-ordering. Conversely: PSA testing "just creates more work' (GP6).

Guidelines UK GPs saw NICE guideline (a clear policy directive) as authoritative, trustworthy, impartia advice against testing; the national guideline influenced practice. "I think people are wary of practicing not in line with that and then they have potential then for criticism" (GP3). The established norm is structured communication with men who ask about testing, using a written information resource.

Mass media and public profile

UK GPs reported that prostate cancer is sometimes in the news media but is "certainly not something which is on the front page of newspapers everyday" (GP8) and "doesn't translate into a lot of men coming and asking

\section{Situation in Australia}

GPs report routinely offering (and encouraging) patients to have multiple tests, perhaps including PSA; healthy patients request health checks regularly. Some considered this 'normal' and/or 'responsible'.

GPs in Australia did not comment much about their medical training and PSA testing; one GP who did said "it's one of the areas where it's pretty much self-taught and you develop your own opinion" (AGP4).

Some Australian GPs had systems to shorten consultations about PSA testing, for example, including PSA in routine bloodwork/'bucket testing', automatic recall so patients could be tested without seeing the GP. Some GPs blamed Australia's fee-for-service health system, which encouraged seeing (and testing) more patients:"it feeds itself to making more diagnoses" (AGP23)

GPs found Australia's competitive information environment about PSA testing hard to navigate: "there's plenty of guidelines, but they're all different and there's nothing official...there's no hard and fast rule" (AGP9). "It's a very tricky area because...opinion is divided depending on who you talk to" (AGP20). Many GPs did not use a guideline, citing patient demand, lack of time, unfamiliarity or a preference for their own judgement; some said RACGP guidance was an unclear 'cop-out'.

Australian GPs said "there has been a lot of media attention to PSA testing over the years" (AGP15), a "part of the big problem with the prostate cancer stuff' (AGP23). Requests increased after media coverage;

\section{Differential effect on PSA testing}

'Screening culture' likely influences default screening practices; in Australia PSA has "become a fairly entrenched part of the male [annual] health check up" (AGP17)

GPs in the UK are specifically trained how to advise asymptomatic men against screening, so seem likely to have more skills to do so, and to default to this practice.

The Australian fee-for-service funding model incentivises [over]servicing; the UK's NHS scarce resources model incentivises caution in creating burden on a limited system.

Having one authoritative guideline seems to encourage consistent practice. At the time of this study, such guidance did not exist in Australia, probably contributing to variation in PSA testing practice. ${ }^{17}$

Conflicting messages and promotion of PSA testing in Australia drives demand from patients; this is absent in the UK. 
Table 2 Continued

Situation in the UK

for PSA tests" (GP1). Many said patients would only hear about PSA testing from their doctor.

Practice protocols All GPs within a single practice in the UK tended to test in a similar way: they "practice as a group and with group supporf' (GP20). This occurred via 'verbal agreement' rather than formal written protocols. Internal practice protocols sometimes permitted practice nurses to PSA-test asymptomatic men without GP involvement.

Method of screening When UK GPs screened (rarely) they often did DRE before or instead of PSA. They though DRE good or standard practice, and valued the information it provided: "the two tests go together" (GP14), "it's a two-part process" (GP23), "doing a PSA alone is worse than doing nothing at all' (GP7). UK urologists reportedly expect GPs to do DRE (although urologists will repeat it).

Referral systems In the UK, referral pathways following particula for men with abnormal results test results are well defined: if PSA was abnormal, GPs would always refer to NHS urology to see the next available (possibly unnamed) consultant, entirely publicly funded. GPs' cancer referrals were audited and GPs made accountable for referrals.

Position taken by urology as a profession
UK GPs said urologists "certainly do not push us to screen men who are otherwise well-if you asked any of them they'll probably say it's actively discouraged' (GP1). (While not reported by these GPs, we know anecdotally and from the literature that some UK urologists have advocated PSA screening.) GPs described close communication and 'strong
Situation in Australia

Differential effect on PSA testing

'media-influenced' patients had preconceived ideas, assuming screening was widely endorsed if sanctioned on TV, "so they see it as their right to have if" (AGP15). "Men know that it's available... so it's hard not to bring it up" (AGP2).

In the absence of Australian consensus guidelines, GPs developed their own testing protocol over years, "I have built up my own idea of practice" (AGP36). Practices need not have formal protocols as "it is a judgement call at the moment' (AGP39).

Australian GPs reported rarely doing DRE in asymptomatic men. Australian GPs were unsure they could detect abnormality via DRE.

After abnormal PSA test results, Australian GPs varied greatly in when, how and to whom they referred. In urban Australia, where there were more urologists, immediate referral after abnormal PSA was common; in rural Australia (fewer urologists) GPs managed abnormal PSA tests for months or years before referral. Australian urologists may be seen publicly or privately; private urology is a competitive marketplace.

Australian GPs said urologists "encouraged PSAs to be done a lot more urgently" (AGP37). Some

GPs accepted this advice; others "politely ignore [d] the advice from urologists in that respect. And from their organizations" (AGP19), as "they have made life very difficult because they're being very unfair on the evidence that's out there" (AGP18). In rural areas, fly-in urologists ran monthly clinics, and influential seminars encouraging testing.
In the UK, DRE was used prior to or instead of PSA, but was not recommended; conversely, until recently Australian guidelines recommended DRE with PSA but it was rarely done.

Presence or absence of protocols at practice level does not seem to explain differences between the two countries: both lacked protocols.

Australia lacked a clear referral pathway for PSA testing, so decisions were made by individual GPs and patients, influenced by a business model of healthcare and a private health sector. In the UK, referral was streamlined and publicly funded.

There was strong variation in GP perceptions and collaborations with urologists, within and between countries. Some Australian GPs were strongly sceptical of some urologists' position; UK GPs were less sceptical. 


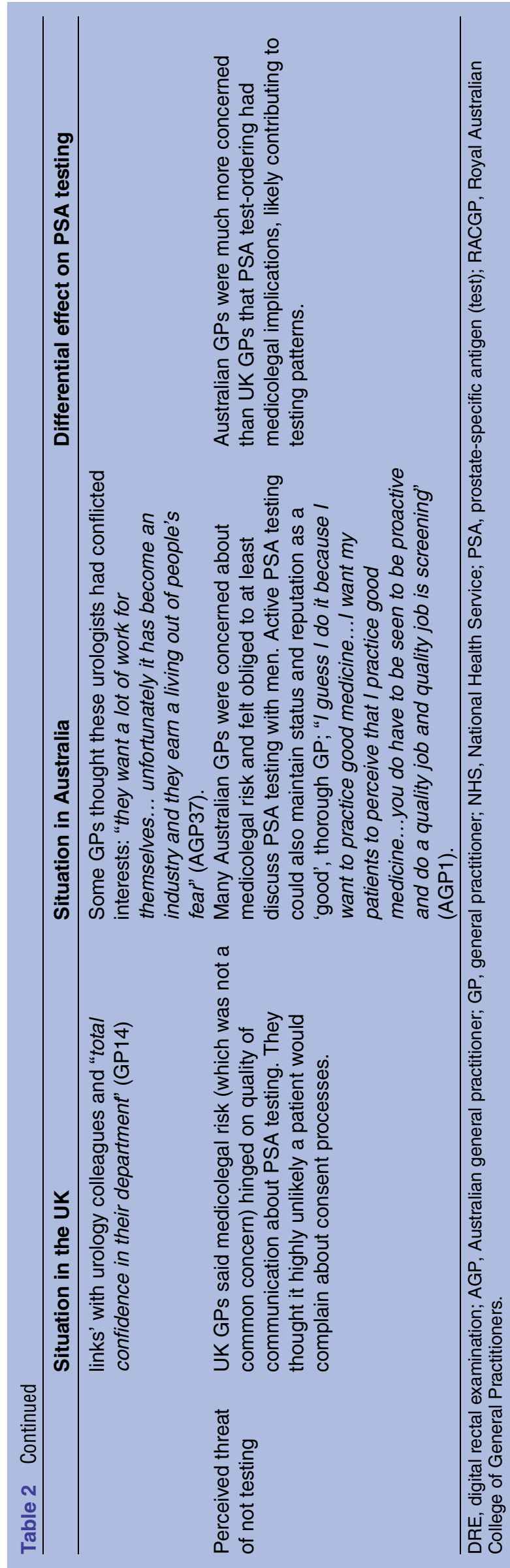

UK GPs reported that the focus of the UK capitation system is on quality of care rather than quantity of care. They described processes in place to support GPs to provide detailed evidence-based information to men who ask about prostate screening; "What happens is you type it [PSA] in and because we have web-based patient data systems, they link...to the patient information stuff, so you tend to use what comes to hand very easily...and because it's online...it's as up to date as it can possibly be" (GP28). Appointments about PSA testing in the UK are (or can be) a two-step process; a 'cooling-off period' is built into usual practice. A policy environment that encourages a two-step process serves to avoid overuse and to ensure decisions are well informed. The UK system looked to be built on an underlying assumption that men who know the facts about PSA testing are less likely to want it. UK GPs reported "feeling quite supported over what we are doing" (GP28) and seemed inclined to operate within the bounds of their health system, while Australian GPs often practiced according to individual standards.

In the UK, referral pathways following particular test results are well defined. GPs reported that the urology departments in their local hospitals had issued referral guidelines for GPs. These were often simplified versions of The National Institute for Health and Care Excellence (NICE) guidance and were seen as prescriptive: as one GP said, "it's a very clear path, it's not a clinical decision" (GP2). Most patients with abnormal PSA results in the UK are referred to a National Health Service (NHS) urology team and see the next available consultant initially on an unnamed basis. It is publicly funded (there are some private urologists, but they are a minority). These urologists therefore do not compete with one another for business to any great extent.

Urologists in Australia serve the public and private sectors: patients can be seen privately or as public outpatients, and private urology, in particular, is a competitive marketplace. The GPs who participated in this study often spoke of patients as consumers, who maintained substantial individual choice in healthcare decisions. Australian GPs reported selecting (with or without the patient) which individual, named urologist a patient will see. They described making this choice based on factors including the 'personalities' of the patient and urologist, and how 'interventionist' they perceived the urologist to be. The GPs varied greatly in when, how and to which specialist they referred abnormal PSA test results. Men could thus potentially receive very different care depending on their GPs approach to PSA testing and urology referral.

\section{GPs appear to rely on different kinds of knowledge in determining their practice}

Accountability, clear expectations set in central policy and support to apply best available evidence meant that UK GPs were equipped to practice in a relatively predictable and standardised way, including when directly asked about PSA testing by their patients. A number of GPs 


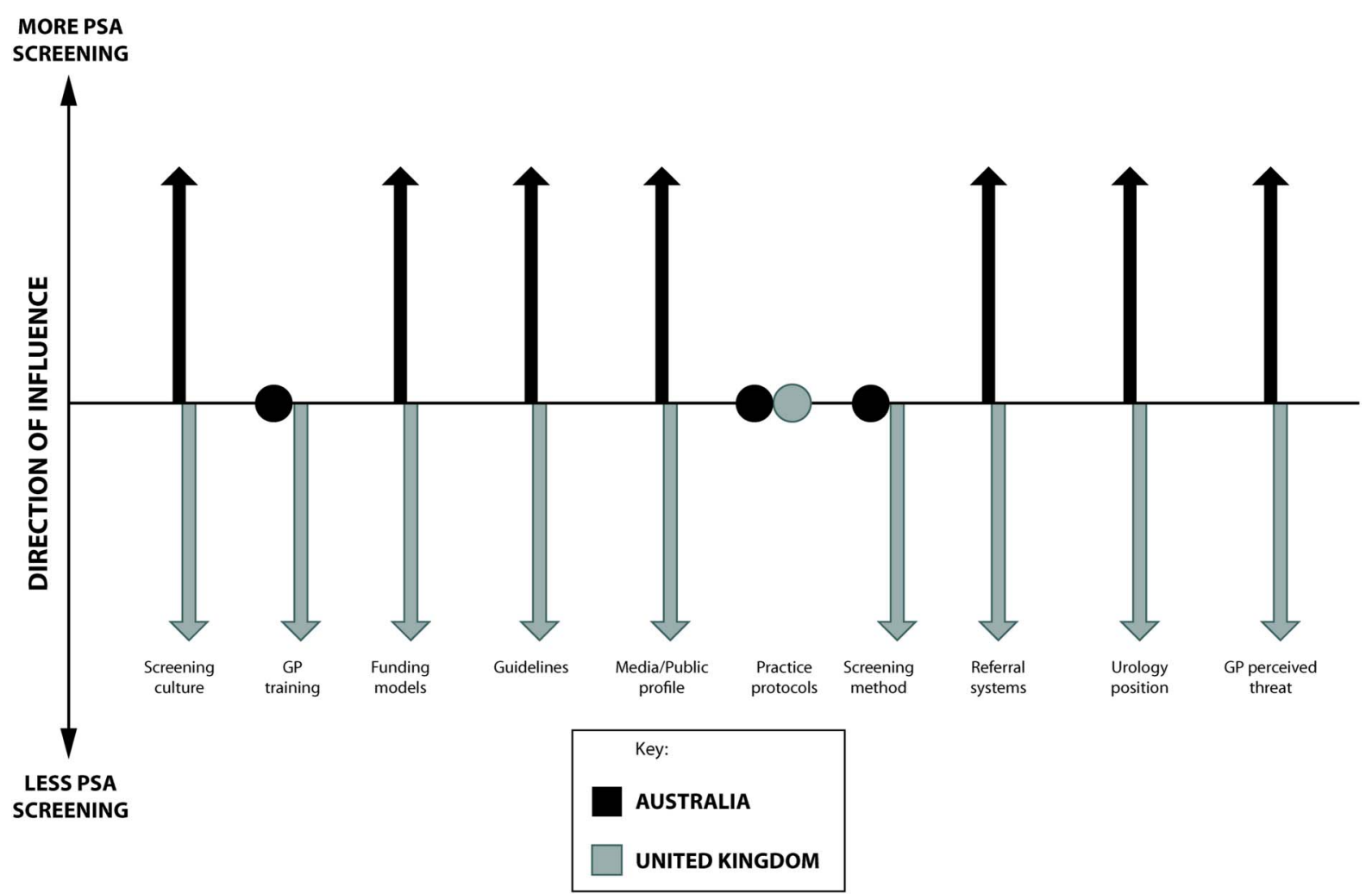

Figure 1 Direction in which system factors described in table 2 drive PSA testing in Australia (black) and the UK (grey). Upward arrows indicate drivers towards more PSA testing, downward arrows indicate drivers towards lower rates of PSA testing, circles indicate neutral factors with no reported impact. PSA, prostate-specific antigen.

from the UK commented throughout the interviews that they had never before reflected on why they approach consultations about PSA testing in the way that they described. They had never really had to grapple with what to do as in regular practice they could confidently follow the available authoritative, evidence-based guidance.

Many GPs in Australia also aimed to practice EBM by following a published guideline. However, there was a proportion who were more likely to practice according to a different idea of evidence: for whom the 'evidence' from their own experience or the experiences of colleagues was critically important in directing their approach to PSA testing. In the Australian context, ambiguity and contestability surround interpretations of the evidence, ${ }^{21}$ and are accompanied by vague professional guidance. Some GPs described research-based directives as incompatible with their day-to-day practice and preferred to deal with what they described as routine cases using their own experience. We reflect on these issues further in the Discussion.

\section{DISCUSSION}

Australia and the UK draw on the same evidence base for prostate cancer screening. The PSA test is in principle available free to eligible men in both countries, and prostate cancer mortality is roughly equivalent. Yet, the rates of PSA testing for prostate cancer risk in the two countries are strikingly different. We identified fundamental differences in how PSA testing occurred and linked this to considerations of how testing was organised. Both countries have factors within their structural and organisational environments that seem to reinforce each other in influencing practice in a particular direction. This combination of multiple factors appears to explain the very different testing and incidence rates, serving mostly to drive more PSA testing in Australia and less PSA testing in the UK.

Australians have been shown empirically to have attitudes broadly in favour of cancer screening. ${ }^{22}$ The Australian media has been shown empirically to deliver a generally pro-PSA-screening message. ${ }^{23}$ These two combined seem likely to increase rather than limit patient demand for PSA testing, and thus to promote rather than retract a market for screening. A fee-for-service payment system allows Australian GPs significant scope to routinely offer PSA testing, and gives them a financial incentive to provide this service to fill the demands of the market. Mixed messages in the current Australian guidelines and some specialists publicly advocating for PSA testing do little to curtail use of the PSA test. In comparison, the medical training of GPs in the UK to avoid PSA testing, strong discouragement from the NHS, little patient demand, limited healthcare resources, and zero financial gains from screening for 
GPs and public sector urologists were reflected in the GPs' explanations of low rates of testing in their respective practices.

\section{What is being done in Australia to address divergent use of the PSA test?}

The provision of and access to PSA testing in Australia is currently extremely heterogeneous, partly dependent on the reasoning and preferences of individual doctors. Newly released national evidence-based clinical guidelines aim to drive greater consistency in testing practices. ${ }^{24}$ The consensus guidelines, which include an 'after the test' component, may prompt more evidencebased discussions and streamlined delivery of consistent information, and standardise referral pathways in Australia.

The authoritative consensus guidelines are a significant move in the right direction for 'smarter screening ${ }^{25}$ in Australia. An accompanying decision aid is being designed to provide an opportunity to make more informed decisions. Yet, the other drivers of screening remain in the Australian setting compared with the UK where PSA screening is discouraged at a system-wide level. The Australian guidance has focused attention primarily on prioritising individual choice. While the new NHMRC guidelines represent a first step in addressing inconsistencies in what GPs are advised to do, and will be a useful information resource to incorporate into evidence-based discussions; they may not suffice to address what is arguably the most important objective: to reduce unnecessary or harmful prostate screening. Although reducing PSA testing rates in Australia is not an explicitly agreed goal of the new NHMRC guidelines, the comparable death rates despite considerably less screening suggest it is likely that the lower rates of PSA testing under the UK system are preferable. In the following section, we suggest areas for consideration and evaluation (alongside the NHMRC guidelines), which may potentially decrease use of the PSA test for screening purposes in Australian primary care.

\section{What can be done in Australia to improve screening practice?}

By comparing Australia with the UK, we have identified features of the context in which screening options are offered that might not otherwise have been appreciated as significant in the Australian setting. Overall, Australian men have fewer practical barriers to undergoing a PSA test, alongside higher incentives for GPs to perform the test. We suggest that, if reducing the rate of PSA testing is a reasonable goal for Australian general practice, the following strategies (structural and organisational) may assist in achieving that goal.

Two-step consultations: Information provision that is separate from PSA testing availability via 'staggered' appointments, which incorporate a cooling-off period. Tambor $e t a l^{26}$ reported that when testing could be obtained conveniently as 'part of a battery of other tests' approach as used in the Australian context, uptake was considerably higher than when additional effort was required to have a test.

Incentivise informed shared decision-making (SDM), as the USA has introduced for lung cancer screening, for example. ${ }^{27}$ UK GPs do not receive reimbursement specifically for engaging in SDM with patients; however, they are accountable for their screening activity and use of NHS resources. GPs in Australia may initially need financial encouragement to implement the NHMRC guidance (provide information rather than offering screening) if this is in contrast to their usual practice. Australia's fee-for-service GP payment system rewards activity in primary care, such as testing, rather than the giving of information to permit an informed choice about whether to test.

Fund PSA testing differently: In the USA, Medicare has considered imposing a penalty for physicians who perform 'non-recommended' prostate cancer screening with the PSA test as part of a federal effort towards valuebased care. ${ }^{28}$ Financial disincentives for GPs and men over time will potentially diminish harm caused by unnecessary screening (eg, of low-risk men or men with a limited life expectancy), as a financial barrier may result in more considered decisions on the part of men and their doctor. However, an ongoing and relevant counterargument is that doctors should not be rewarded for withholding a test that could help some men, nor restrict the options of men with limited financial means. Welch and others have suggested introducing a small cost to men for PSA testing. ${ }^{29}$ Introducing a small cost for a PSA test with clearly communicated exceptions (eg, men with strong risk factors for prostate cancer) may be a reasonable option in Australia. There are plans in Australia to consider changes to the Medicare Benefits Schedule item number to align with the new PSA testing guidelines and only allow coverage for a PSA test every second year, rather than annually. However, the PSA test is currently in principle available free to men in Australia and the UK, suggesting cost may not be a key factor influencing more frequent testing in Australia. This would be a pertinent topic for further research.

\section{Why might achieving a shift in GP practice be difficult?}

Although the varied structural and organisational conditions in the respective healthcare systems of Australia and the UK seemed to explain much of the difference in GP accounts of their PSA testing practice-at the patient and consultation level-another layer to the decision-making environment was also evident. Gabbay and le May's theory of 'mindlines' is particularly relevant to our analysis. We hypothesise from our data that GPs from Australia and the UK are following different 'mindlines', shaped by their respective cultures, contexts and experiential knowledge.

We propose that UK GPs have internalised an organisationally embedded consistent mindline, based on the evidence-based recommendations from a trusted 
authoritative voice. All GPs appeared to agree about what practice was appropriate, implementing a relatively similar version of evidence-based practice. There was less room to move in their individual interpretations of the evidence because the professional guidance was consistent and not contested-UK GPs were advised which evidence was appropriate, and provided with supporting materials to distribute to men. Funding arrangements also put explicit boundaries around what is considered acceptable practice. The mindline used by UK GPs seems to have developed during their training and was subsequently collectively shared and reinforced via reliable professional networks, including urologists. It was rarely challenged because of clear communication, a collective understanding of requirements and expectations, and limited exposure to men requesting prostate screening.

We suggest that, in contrast to the UK, GPs in Australia are accustomed to a noisy marketplace of conflicting advice, including from urologists. As a result, their mindlines appear to be more independently constructed based on individual experiences, and strongly influenced by contextual considerations. Like the UK GPs, the Australian GPs' mindlines were developed and reinforced via similar processes of experience, repetition and interactions, but these occurred within local circumstances and macro systems that looked very different to the UK situation. It is likely that Australian GPs' mindlines vary considerably from one GP to the next as they navigate and interpret the changeable conditions. Australian GPs described a broad spectrum of men regularly asking about PSA screening, each with very different expectations. So, while an individual Australian GP may have a relatively unified approach to PSA screening decisions and discussions, specific contexts and patient presentations could prompt temporary deviation from this mindline in favour of an alternative.

While producing guidelines (such as the NHMRC guideline) as a formal source of knowledge is essential, against a background of clinicians' diverse ingrained habits and history, guidelines are unlikely to be enough to alter established clinical practice in Australian GPs. Nonetheless, consistent guidelines provide a much needed foundation to build on. As Gabbay and le May conclude, practitioners have a collective responsibility to ensure their mindlines are based on research evidence wherever possible, ${ }^{15}$ and the NHMRC guidelines provide a consensus regarding the appropriate evidence base. However, above all, our research has demonstrated the overwhelming influence of local contexts on clinical practice. GPs' established mindlines and rules of thumb appear to interact with the social and organisational context. If policy leaders want to promote practice consistent with the consensus guidelines, they are likely to need to work actively, alongside providing ongoing support for GPs, in directing how the NHMRG guidelines are received, implemented and used in practice. The process should prioritise establishing GPs' trust in the guidelines as a reliable source of information for supporting modified practice patterns among those whose practice is not aligned with the new recommendations. Our findings and Gabbay and le May's theory suggest that guideline dissemination might be best targeted through favoured sources of information, including influential GP and specialist colleagues-although conflicts of interest might make this challenging.

Future research might usefully explore the likelihood and feasibility of a cultural shift around PSA screening, examining men's and broader public enthusiasm to screen in Australia. Schwartz et $a l^{30}$ found that the majority of American men they sampled would over-rule their physician's recommendations for less frequent or no screening. A closer look at urologists' 'mindlines' may also be relevant, as these appear to influence the mindlines of GPs.

\section{Limitations}

We interviewed a large number of highly informative participants (GPs) with diverse opinions and approaches; participation in the study was self-selecting. It is possible that physicians with particularly strong opinions about prostate cancer screening were more likely to volunteer.

\section{CONCLUSION}

Important drivers of prostate cancer screening are evident at a number of levels. PSA testing rates arise from more than individual GP attitudes towards screening: current and historical structures, systems and rules all play a significant role in creating the mindlines that GPs employ in their practice. It is likely that all of these influences will need to be addressed if these mindlines, and thus practice, are to change.

Twitter Follow Kristen Pickles @PicklesKristen, Vikki Entwistle @entwistlev, Stacy Carter @stacymcarter and Lucie Rychetnik @LucieRychetnik

Contributors All authors conceived the study and were involved in designing the study and developing the methods. SMC and LR obtained funding and are Cls on the NHMRC-funded project grant. KP conducted the interviews, had full access to all data in the study, and takes responsibility for the integrity of the data and the accuracy of the data analysis. KP drafted the manuscript. All authors contributed to the interpretation of the analysis and critically revised the manuscript.

Funding The project was funded by NHMRC grant number 1023197. SC was supported by NHMRC Career Development Fellowship number 1032963 when this work was completed.

Disclaimer The funders had no role in the design or conduct of the study; in the collection, analysis, and interpretation of the data; or in the preparation or approval of the manuscript.

Competing interests All authors have completed the ICMJE uniform disclosure form at http://www.icmje.org/coi_disclosure.pdf and declare: SC was supported by NHMRC Career Development Fellowship number 1032963 when this work was completed; they have no financial relationships with any organisations that might have an interest in the submitted work in the previous 3 years; they have no other relationships or activities that could appear to have influenced the submitted work. 
Ethics approval All study procedures were approved by the Cancer Institute New South Wales and the University of Sydney Human Research Ethics Committee [\#15245]. Each participant had an opportunity to discuss the study, and gave written consent prior to participation.

Provenance and peer review Not commissioned; externally peer reviewed.

Data sharing statement No additional data are available.

Transparency KP affirms that the manuscript is an honest, accurate, and transparent account of the study being reported; no important aspects of the study have been omitted

Open Access This is an Open Access article distributed in accordance with the Creative Commons Attribution Non Commercial (CC BY-NC 4.0) license, which permits others to distribute, remix, adapt, build upon this work noncommercially, and license their derivative works on different terms, provided the original work is properly cited and the use is non-commercial. See: http:// creativecommons.org/licenses/by-nc/4.0/

\section{REFERENCES}

1. Carter HB, Albertsen PC, Barry MJ, et al. Early detection of prostate cancer: AUA Guideline. J Urol 2013;190:419-26.

2. Mistry M, Parkin DM, Ahmad AS, et al. Cancer incidence in the United Kingdom: projections to the year 2030. Br J Cancer 2011;105:1795-803.

3. Globocan 2012: estimated cancer incidence, mortality, and prevalence worldwide in 2012: International Agency for Research on Cancer. World Health Organisation, 2012. http://globocan.iarc.fr/ Pages/fact sheets cancer.aspx

4. Williams N, Hughes LJ, Turner EL, et al. Prostate-specific antigen testing rates remain low in UK general practice: a cross-sectional study in six English cities. BJU Int 2011;108:1402-8.

5. Moss S, Melia J, Sutton J, et al. Prostate-specific antigen testing rates and referral patterns from general practice data in England. Int J Clin Pract 2016;70:312-18.

6. Medicare Benefits Schedule Book Category 6: Australian Government Department of Health; 2014. Department of Human Services, Commonwealth of Australia. http://wiki.cancer.org.au/ australia/Guidelines:PSA_Testing/Introduction\#cite_note-Citation: Australian Government_Department of Health_2014-16

7. Trevena JA, Rogers KD, Jorm LR, et al. Quantifying under-reporting of pathology tests in Medical Benefits Schedule claims data. Aust Health Rev 2013;37:649-53.

8. Holden CA, McLachlan RI, Pitts M, et al. Men in Australia Telephone Survey (MATeS): a national survey of the reproductive health and concerns of middle-aged and older Australian men. Lancet 2005;366:218-24

9. Baade PD, Youlden DR, Coory MD, et al. Urban-rural differences in prostate cancer outcomes in Australia: what has changed. Med J Aust 2011;194:293-6.

10. Young AF, Dobson AJ. The decline in bulk-billing and increase in out-of-pocket costs for general practice consultations in rural areas of Australia, 1995-2001. Med J of Aust 2003;178:122-6.

11. De Abreu Lourenco R, Kenny $P$, et al. Factors affecting general practitioner charges and Medicare bulk-billing: results of a survey of Australians. Med J Aust 2015;202:87-90.

12. Jones G, Savage E, Hall J. Pricing of general practice in Australia: some recent proposals to reform Medicare. J Health Serv Res Policy 2004;9(Suppl 2):63-8.
13. Weber MF, Cunich M, Smith DP, et al. Sociodemographic and health-related predictors of self-reported mammogram, faecal occult blood test and prostate specific antigen test use in a large Australian study. BMC Public Health 2013;13:429.

14. Gabbay J, le May A. Practice-based evidence for healthcare: clinical mindlines. Abingdon, Oxon: Routledge, 2011.

15. Gabbay J, le May A. Evidence based guidelines or collectively constructed "mindlines?" Ethnographic study of knowledge management in primary care. BMJ 2004;329:1013.

16. Bryant A, Charmaz K. The Sage handbook of grounded theory. Sage, 2007

17. Pickles K, Carter SM, Rychetnik L. Doctors' approaches to PSA testing and overdiagnosis in primary healthcare: a qualitative study. BMJ Open 2015;5:e006367.

18. Entwistle VA, Watt IS, Davis $\mathrm{H}$, et al. Developing information materials to present the findings of technology assessments to consumers: the experience of the NHS Centre for Reviews and Dissemination. Int J Technol Assess Health Care 1998; 14:47-70.

19. Prostate cancer screening: summary of the review prepared by the Australian Health Technology Advisory Committee. Canberra: Department of Health and Community Services; 1996.

20. Australian Cancer Society. Prostate cancer screening: guidelines for health professionals. Cancer Forum 1995;19:47-50.

21. Pickles K, Carter SM, Rychetnik L, et al. General practitioners' experiences of, and responses to, uncertainty in prostate cancer screening: insights from a qualitative study. PLOS ONE 2016;11: e0153299.

22. Livingston $\mathrm{P}$, Wakefield M, Elwood JM. Community attitudes towards the early detection of cancer in Victoria, Australia. Aust N Z J Public Health 2007;31:26-9.

23. MacKenzie R, Chapman S, Barratt A, et al. "The news is [not] all good": misrepresentations and inaccuracies in Australian news media reports on prostate cancer screening. Med J Aust 2007; $187: 507-10$.

24. Clinical practice guidelines PSA testing and early management of test-detected prostate cancer. Sydney: Cancer Council Australia 2016. Prostate Cancer Foundation of Australia and Cancer Council Australia PSA Testing Guidelines Expert Advisory Panel. Clinical practice guidelines PSA Testing and Early Management of TestDetected Prostate Cancer. Sydney: Cancer Council Australia. http://wiki.cancer.org.au/australiawiki/index.php?oldid=122816 (accessed 24 Nov 2016)

25. Gulati R, Gore JL, Etzioni R. Comparative effectiveness of alternative prostate-specific antigen-based prostate cancer screening strategies. Ann Intern Med 2013;158:145-53.

26. Tambor ES, Bernhardt BA, Chase GA, et al. Offering cystic fibrosis carrier screening to an $\mathrm{HMO}$ population: factors associated with utilization. Am J Hum Genet 1994;55:626-37.

27. Decision memo for screening for lung cancer with low dose computed tomography (LDCT). Centers for Medicare \& Medicaid Services. https://www.cms.gov/medicare-coverage-database/details/ nca-decision-memo.aspx?NCAld=274

28. Beck M. Doctors could be penalized for ordering prostate tests: The Wall Street Journal, 2015. http://www.wsj.com/article email/doctorscould-be-penalized-for-ordering-prostate-tests-1447993920IMyQjAxMTE1MzI3MDkyNDA1Wj

29. Welch HA, Peter C. Why doctors shouldn't be punished for giving prostate tests: The New York Times; 7 January 2016. http://www. nytimes.com/2016/01/07/opinion/why-doctors-shouldnt-be-punishedfor-giving-prostate-tests.html? $r=2$

30. Schwartz LM, Woloshin S, Fowler FJ Jr, et al. Enthusiasm for cancer screening in the United States. JAMA 2004;291:71-8. 